\title{
Detection of the Triclorfon Base on Bioluminescence Resonance Transfer among Functional CdZnSe/ZnS Quantum Dots
}

Hai $\mathrm{NN}^{*}$, Nga $\mathrm{PT}^{2}$, and Cao $\mathrm{DT}^{2}$

${ }^{1}$ Department of Education, Quangninh Province, Vietnam

${ }^{2}$ Institute of Materials Science, Vietnam Academy of Science and Technology, 18, Hoangquocviet Road, Caugiay

District, Hanoi, Vietnam

${ }^{*}$ Corresponding author: Hai NN, Department of Education, Quangninh Province, Vietnam, E-mail: haidhsphn@gmail.com

Citation: Hai NN, Nga PT, Cao DT (2018) Detection of the triclorfon base on bioluminescence resonance transfer among functional CdZnSe/ZnS quantum dots. J Mater Sci Nanotechnol 6(2): 203

Received Date: February 26, 2018 Accepted Date: April 12, 2018 Published Date: April 18, 2018

\begin{abstract}
The synthesis of ternary quantum dots (TQDs) have a significant effect on the use of nano scale crystals in biology, photonics and photovoltaic. Rather than controlling the size of TQDs, making three-component TQDs is also an approach to changing the energy of their barrier width, typically using three-component substances such as $\mathrm{Zn}_{\mathrm{x}} \mathrm{Cd}_{1-\mathrm{x}} \mathrm{Se}$. In addition, wrapping the TQDs core with the shell of higher band gap $(\mathrm{ZnS})$ can further trap the electrons $(e)$ and holes $(h)^{\mathrm{x}}$ and enhance the emission intensity. Dispersed TQDs have been used with the carboxyl group $\mathrm{COOH}$ - and bonded to the streptavidin (SA) molecule by electrostatic interaction, which is one of the strongest interactions between biological molecules, then the human acetyl cholinesterase enzyme (AChE) is linked to SA by avidin-biotin binding. The substance to be analyzed is the active molecules of trichlorfon pesticides (organic phosphate group). It has a toxic mechanism that inhibits the activity of the enzyme AChE. With the bioluminescence resonance energy transfer (BRET), TQDs act as acceptors and the surrounding proteins are the donors, as the AChE is tied around the TQDs. In particular, BRET depends on the distance between donor and acceptor rather than the ratio of quantity. The normal distance for this resonance shift is between 2 and $9 \mathrm{~nm}$.
\end{abstract}

Keywords: Quantum Dots; CdZnSe; Bioluminescence; Pesticides

\section{Introduction}

Organophosphorus (OP) compound is widely used in plant protection for insect killing, easy synthesis, and low cost. The mentioned pesticide residues are harmful to the environment, human health, and animals through nervous system poisoning by inhibiting the activity of $\mathrm{AChE}$, the most important enzyme involved in nerve transmission [1,2]. The principal role of AChE is terminating nerve impulse transmission at cholinergic synapses by rapid hydrolysis of the neurotransmitter acetylcholine (ACh) each molecule of AChE degrades about 25000 molecules of ACh per second, leading to in vivo accumulation of ACh, resulting in serious impairment on nerve functions [3].

Instead of controlling the size of TQDs, the formation of ternary alloy quantum dots with $\mathrm{Zn}_{\mathrm{x}} \mathrm{Cd} \mathrm{f}_{1-\mathrm{x}} \mathrm{Se} / \mathrm{ZnS}$ core/shell structure takes an alternative approach to tune their energy band gaps, typically using ternary or quaternary compositions. In these multicompositional TQDs, the band gap energy is determined by the combination of their chemical stoichiometry and particle size. The TQDs could be achieved by changing the constituent cation/anion precursor amounts. The widely tuneable emissions of Cd $\mathrm{Zn} \mathrm{n}_{1-\mathrm{x}} \mathrm{Se}$ quantum dots is in the wavelength range from 534 to $620 \mathrm{~nm}[2]$.

Here we present the results of extensive research on CdZnSe/ZnS TQDs for detecting trichlorfon (TF) - one type of organophosphorus pesticide (OP). AChE acts as the indicator and is determined indirectly by using acetylthiocholine (ATCh) which is a very strong hydrolyte in the presence of ATCh and used as an indicator for the activity [4].

The system of TQDs-AChE was built based on the electrostatic and biological interactions (by mercaptopropionic acid - MPA and streptavidin - SA), illustrated in Figure 1. 


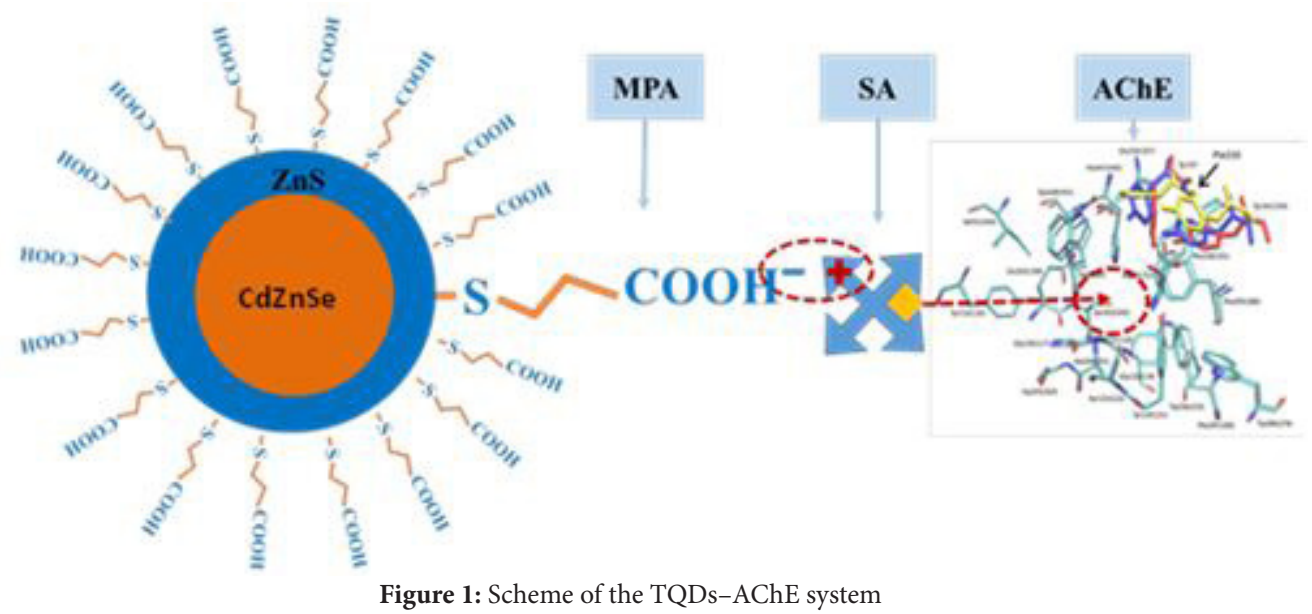

\section{Experimental}

The TQDs cores were synthesized with a constant precursor ratio of $\mathrm{Cd}_{02} \mathrm{Zn}_{0.8} \mathrm{Se}$. This method was adapted from an existing work for CdSe quantum dots [5,6]. We used ultra-pure nitrogen flow for all synthetic routes. The molar ratio of the precursors for a typical synthesis of $0.5 \mathrm{mM} \mathrm{Cd}_{02} \mathrm{Zn}_{0.8}$ Se alloy nanocrystal QDs are $\mathrm{Cd} / \mathrm{Zn} / \mathrm{Se}=0.2 / 0.8 / 3.33 ; \mathrm{TOPO} / \mathrm{HDA}=55 / 45$. First, we injected TOP-Se precursor at $100{ }^{\circ} \mathrm{C}$ and heated it up to $190^{\circ} \mathrm{C}$. After injecting TOP-Zn, the temperature was raised to $270{ }^{\circ} \mathrm{C}$ and TOP$\mathrm{Cd}$ precursor was injected. The nucleation of CdZnSe TQDs cores started shaping quickly at $260^{\circ} \mathrm{C}$. After 20 minutes at $280-290$ ${ }^{\circ} \mathrm{C}$, we obtained $\mathrm{Cd}_{0.2} \mathrm{Zn}_{0.8}$ Se alloy QD core. Second, was the synthesis of a $\mathrm{ZnS}$ shell on the $\mathrm{Cd}_{0.2} \mathrm{Zn}_{0.8}$ Se core. We evacuated the air from the mixture of $0.25 \mathrm{mmol} \mathrm{CdZnSe}$ TQDs, TOPO and HDA for an hour at $50^{\circ} \mathrm{C}$. The shell growth processed at $220^{\circ} \mathrm{C}$ and the amount of $\mathrm{S}$ was $0.115 \mathrm{ml}[5,7,8]$.

The AChE enzymes (EC 3.1.1.7, activity unit: $500 \mathrm{U} / 0.7 \mathrm{mg}$ ) and S-acetylthiocholine iodide (ATChI) were purchased from SigmaAldrich; ATCh was prepared from ATChI, and trichlorfon (TF) were obtained from Vietnam Plant Protection Department (PPD) $[2,6]$. The TQDs were surface-modified by MPA in order to assure water solubility and SA molecules were attached to the TQDs [7]. Base on coulomb interaction, the AChE was conjugated to form the system of TQDs - AChE. TQDs-SA and AChE were mixed and incubated at $37^{\circ} \mathrm{C}$ for 10 minutes. Different amounts of the pesticides were added to the same corresponding amount of ATCh $(2 \mu \mathrm{mol})$, and the mixture was added to the biosensor solution, incubated at $37^{\circ} \mathrm{C}$ for $20 \mathrm{~min}$. The $\mathrm{pH}$ values of the initial solutions were around 7.1-7.3. The optical properties of the TQDs were determined by the transmission electron microscopy (TEM-JEOL Jem 1010 microscope system). Photoluminescence (PL) measurements with laser excitation at $325 \mathrm{~nm}$ and $442 \mathrm{~nm}$ have been done.

\section{Results and Discussion}

TEM images of $\mathrm{Cd}_{0.2} \mathrm{Zn}_{0.8}$ Se TQDs illustrated in Figure 2 show their quasi-spherical shape. The average sizes are measured to be about 3.4-5 $\mathrm{nm}$ and about 5.2-7.0 $\mathrm{nm}$ for the core TQDs grown for $20 \mathrm{~min}$ and the core/shell TQDs, respectively. These core/shell TQDs create a large surface area for conjugating of biology agents [9].

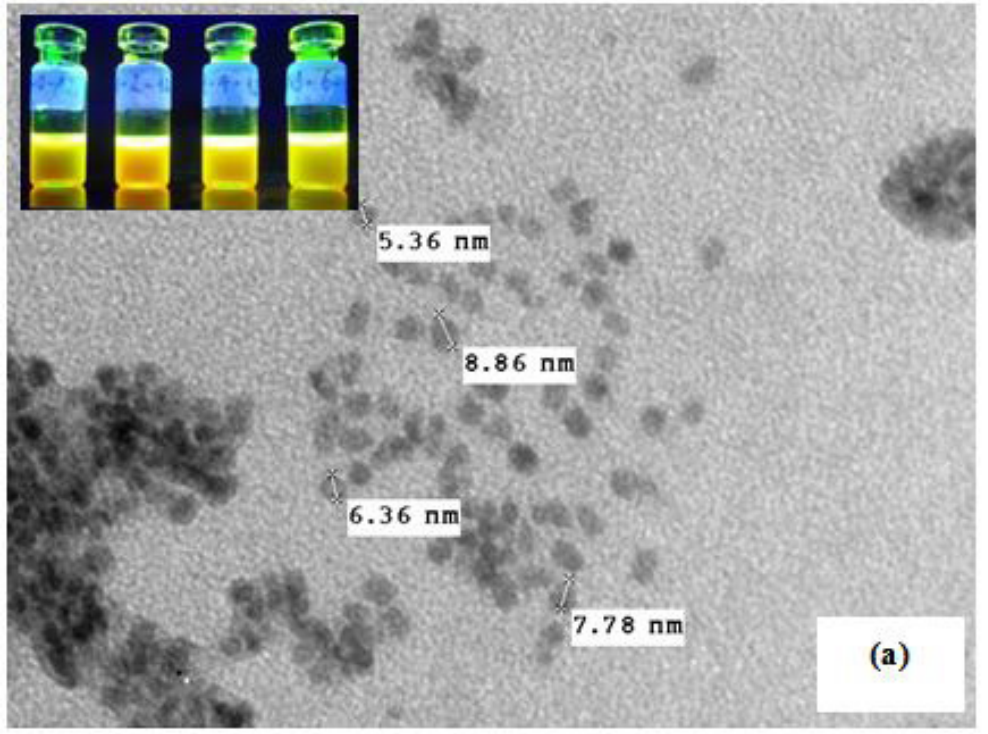




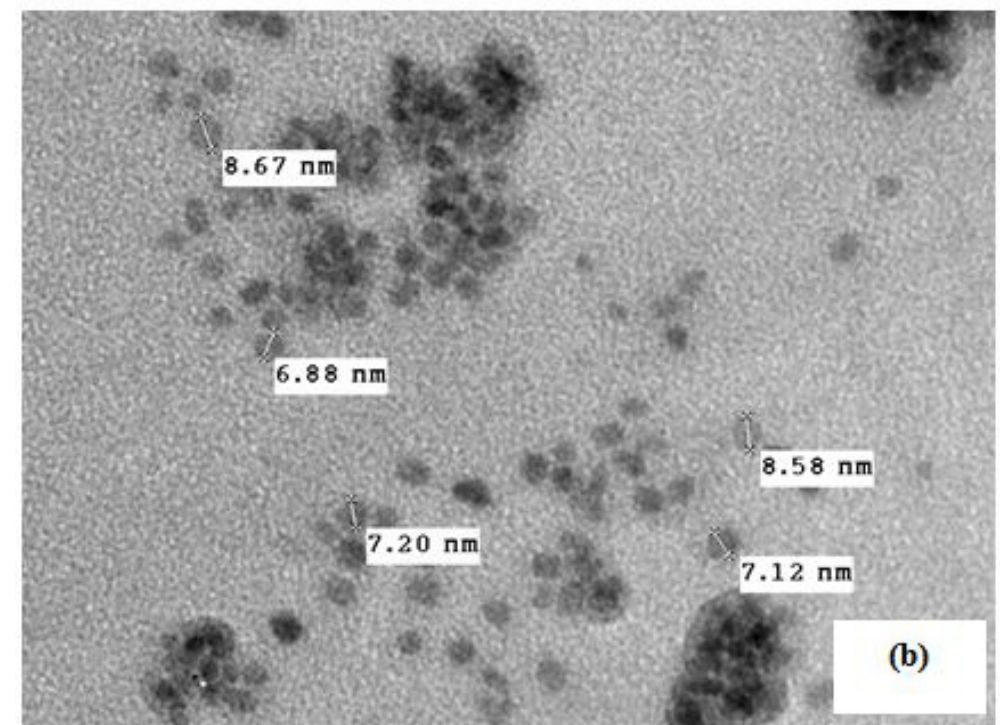

Figure 2: STEM images of $\mathrm{Cd}_{0,2} \mathrm{Zn}_{0,8}$ Se/ZnS 2 monolayer (a) and $\mathrm{Cd}_{0,2} \mathrm{Zn}_{0,8} \mathrm{Se} / \mathrm{ZnS} 4$ monolayer (b)

The composition of the TQDs also measured by EDS (not shown here) indicates that subsequent incorporation of zinc into the crystal lattice takes place after reaction time exceeds $18 \mathrm{~min}$ and that the formation of $\mathrm{Cd}_{0.2} \mathrm{Zn}_{0.8}$ Se nanocrystals is observed. Elemental analysis of the TQDs reveals the presence of Zn, Cd, and Se in the TQDs core and the presence of the Zn and S elements in the shell.

Figure 3 shows the absorption and photoluminescence (PL) spectra of CdZnSe/ZnS. The PL spectrum under the excitation wavelength of $442 \mathrm{~nm}$ exhibits an emission peak at $568 \mathrm{~nm}$ with FWHM of about $34.4 \mathrm{~nm}$. The excitonic absorption peak at 556 $\mathrm{nm}$ and the strong PL peak at $568 \mathrm{~nm}$ of TQDs show the eligible optical properties to build biosensor.

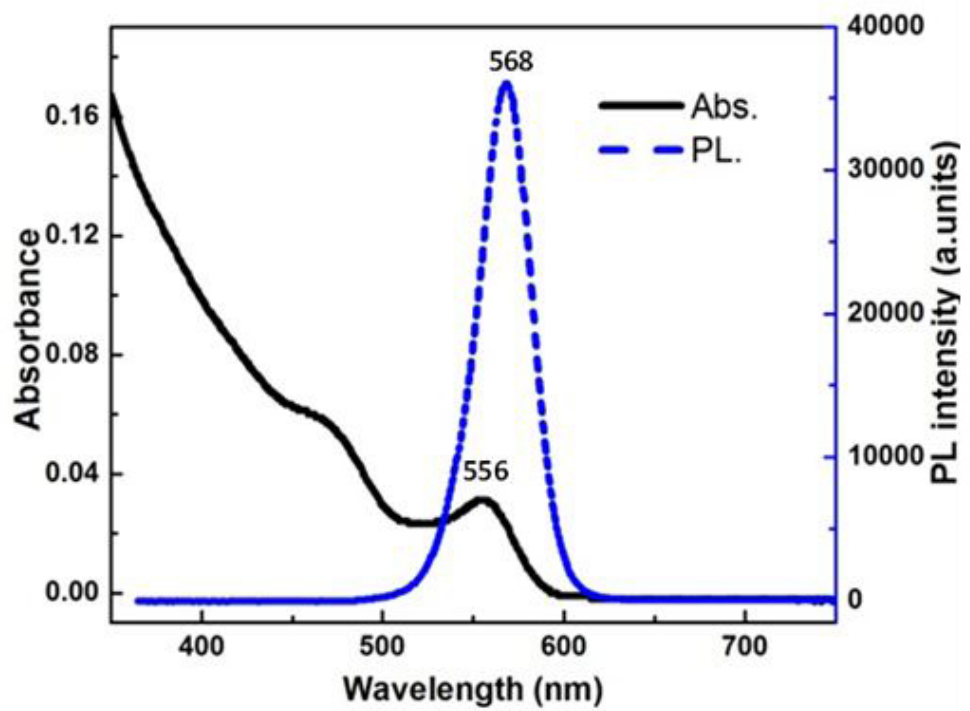

Figure 3: Absorption (solid line) and PL (dotted line) spectra of CdZnSe/ZnS quantum dots

Moreover, the criteria were minimizing blinking effect. This directly affects the "in" and "out" signals of the TQDs in the system. One third of the CdZnSe/ZnS 4-monolayer samples have the traces and the blinking is 35\% in the on time state [10,11]. In this system, ATCh acts as an indicator for the presence of the AChE enzymatic activity, while the TQDs act as the luminescence indicators for the hydrolysis of ATCh. Eventually, the luminescence from TQDs can indicate the AChE enzymatic activity or correspondingly the pesticide content because of pesticide being added into the mixed solution. The pesticide would bind and inhibit the AChE enzymatic activity.

AChE catalyses the hydrolysis of ATCh to produce TCh, which bears an additional thiol group ( $-\mathrm{SH}$ ) to possibly decrease the $\mathrm{pH}$ surrounding the TQDs (Figure 1). It was calculated with the amount of $100 \mu \mathrm{l}$ TQDs, $122 \mu \mathrm{l}$ AChE and $16.7 \mu \mathrm{l}$ ATCh, we observed the PL intensity of TQDs/AChE/ATCh solution decreased, and it took $12 \mathrm{~min}$ to reach the stable value (shown in Figure $5 \mathrm{a}$ ). The solution of ATCh/TF with concentration of $2 \mathrm{ppm}$ was mixed separately from the TQDs/AChE. The integrated PL intensity was measured to be increasing 1.75 times for 26 min, illustrated in Figure 5b. 


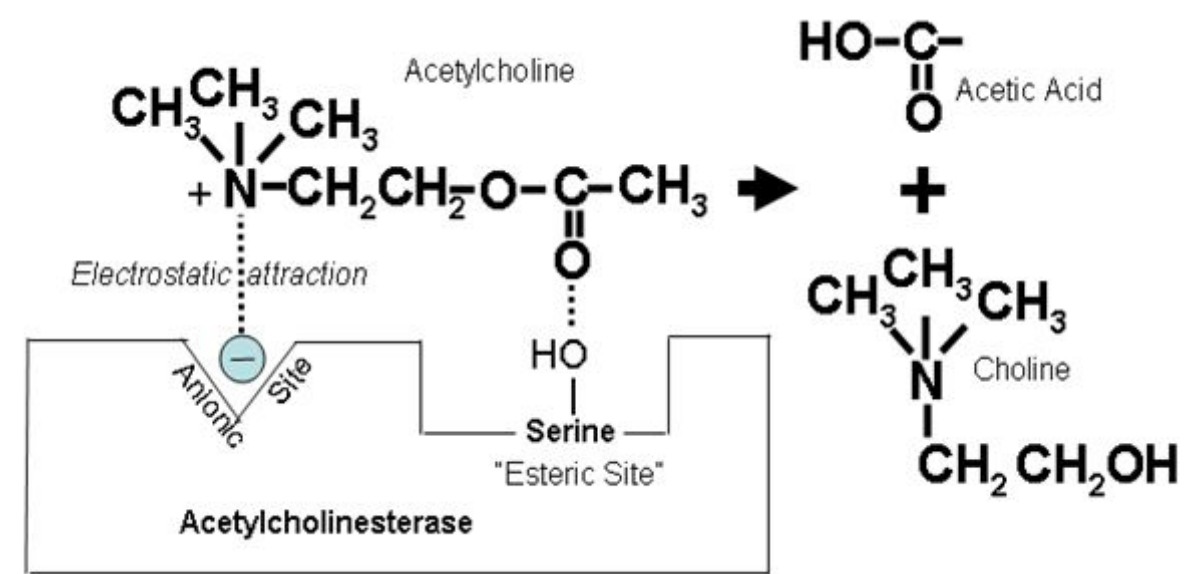

Figure 4: Scheme of general chemical structures for the most popular categories of AChE inhibitors, namely organophosphates
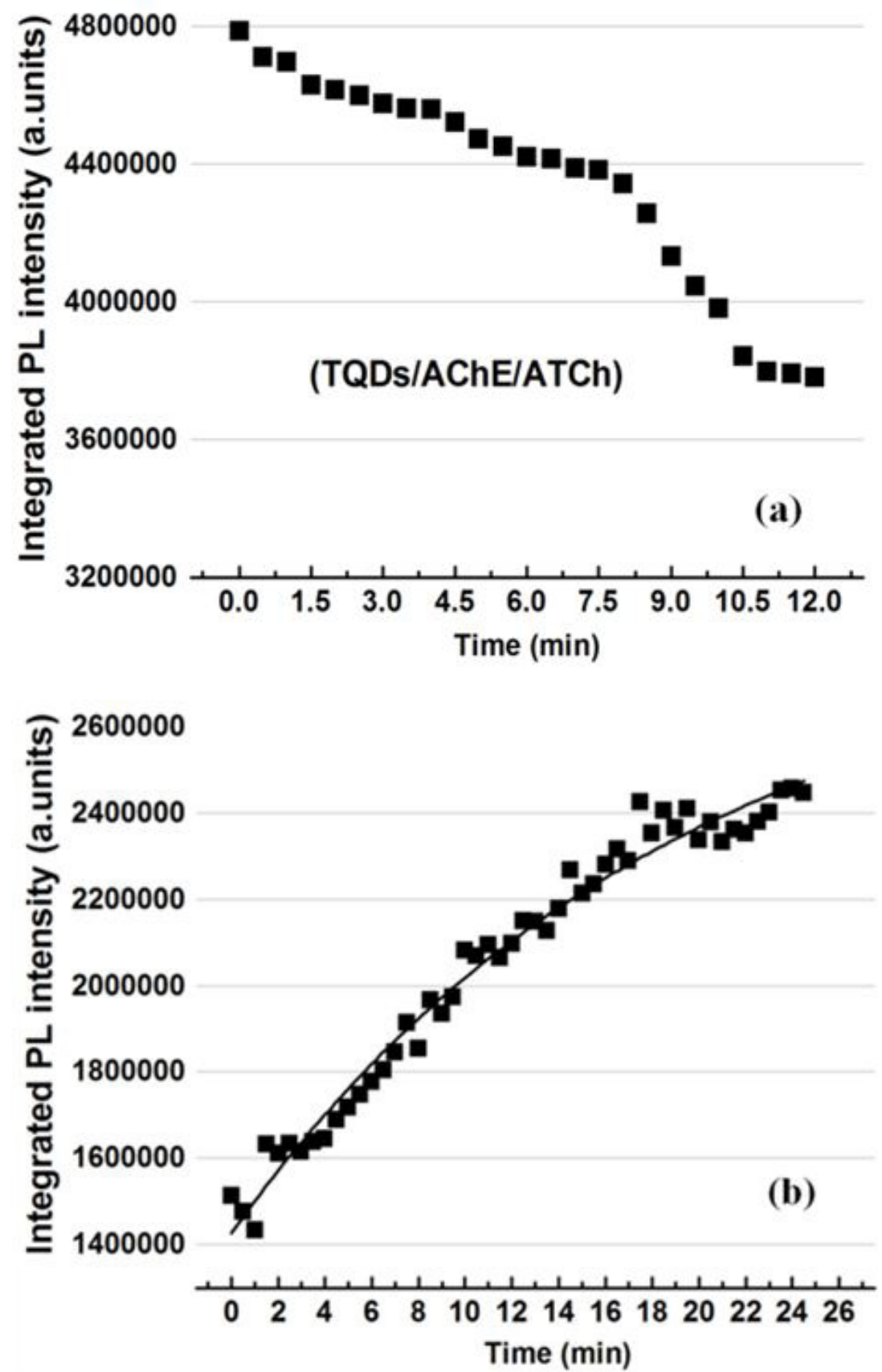

Figure 5: Changes in PL intensity over time of TQDs/AChE/ATCh (a) and TQDs/AChE - ATCh/TF (b) 
Figure 6 presents the same trend of PL intensity increasing with concentration at $0.05 ; 2.0 ; 5.0 ; 8.0$ and 10.0 ppm. (The European standard is $0.05 \mathrm{ppm}$ for most agricultural products) [12]. The $\mathrm{pH}$ values were measured at the temperature of $31.5-32.0{ }^{\circ} \mathrm{C}$. The minimum $\mathrm{pH}$ value of TQDs/AChE/ATCh was about 5.51 and raised with increasing concentrations of trichlorfon until reaching the maximum $\mathrm{pH}$ value of the mixture TQDs/AChE initially about 7.11-7.22. The PL intensity (I) of the corresponding concentrations is compared to that of TQDs/AChE/ATCh (Io); the maximum ratio is 1.52 for the case of trichlorfon at concentration of $5.0 \mathrm{ppm}$. (Table 1).

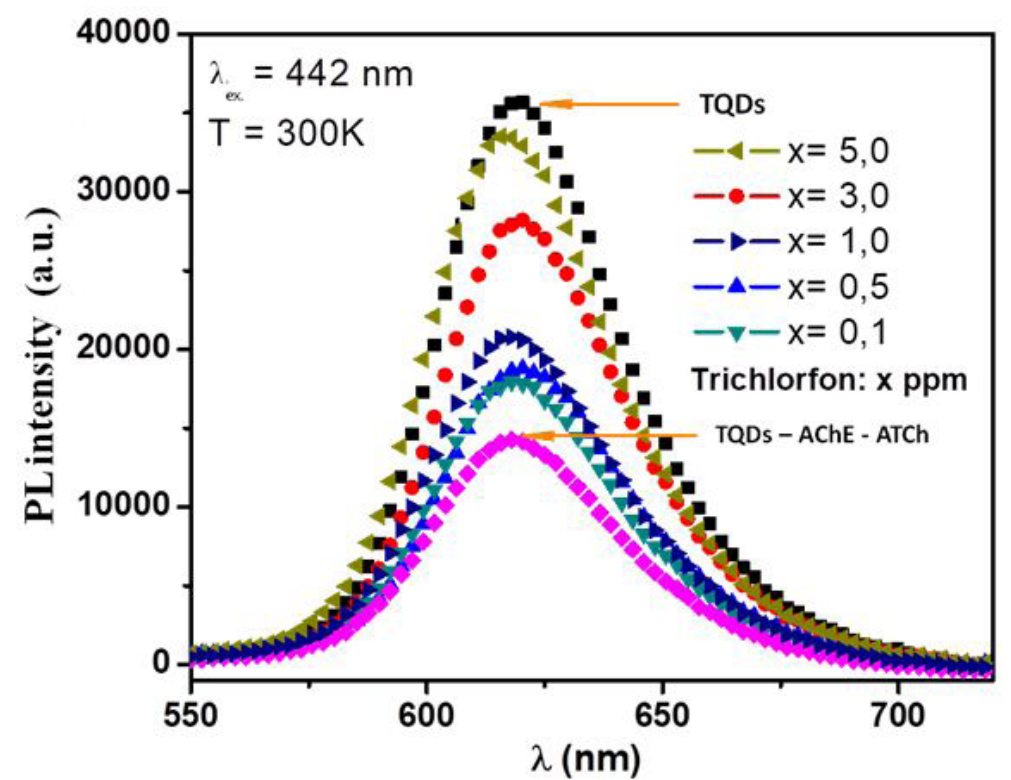

Figure 6: Fluorescence spectra of TQDs-AChE with ' $\mathrm{x}$ ' ppm of trichlorfon $(\mathrm{x}=0.1 ; 0.5 ; 1.0 ; 3.0 ; 5.0)$

The MPA-capped core/shell TQDs are highly sensitive to $\mathrm{pH}$, with greater fluorescence at higher $\mathrm{pH}$, and a small red shift in the peak emission with increasing $\mathrm{pH}$ [8]. This increase of fluorescence intensity with increasing $\mathrm{pH}$ has also been reported for CdSe/ ZnS binary quantum dots [13].

Figure 7 shows that the catalytic triad is composed of Glu334, His447 and Ser203. Soman is covalently bound to Ser203 with the pinacolyl tail interacting with Trp86 (choline binding site) and with the phosphate oxygen forming electrostatic interactions with Gly121 and Gly122 (oxyanionic hole) [15].

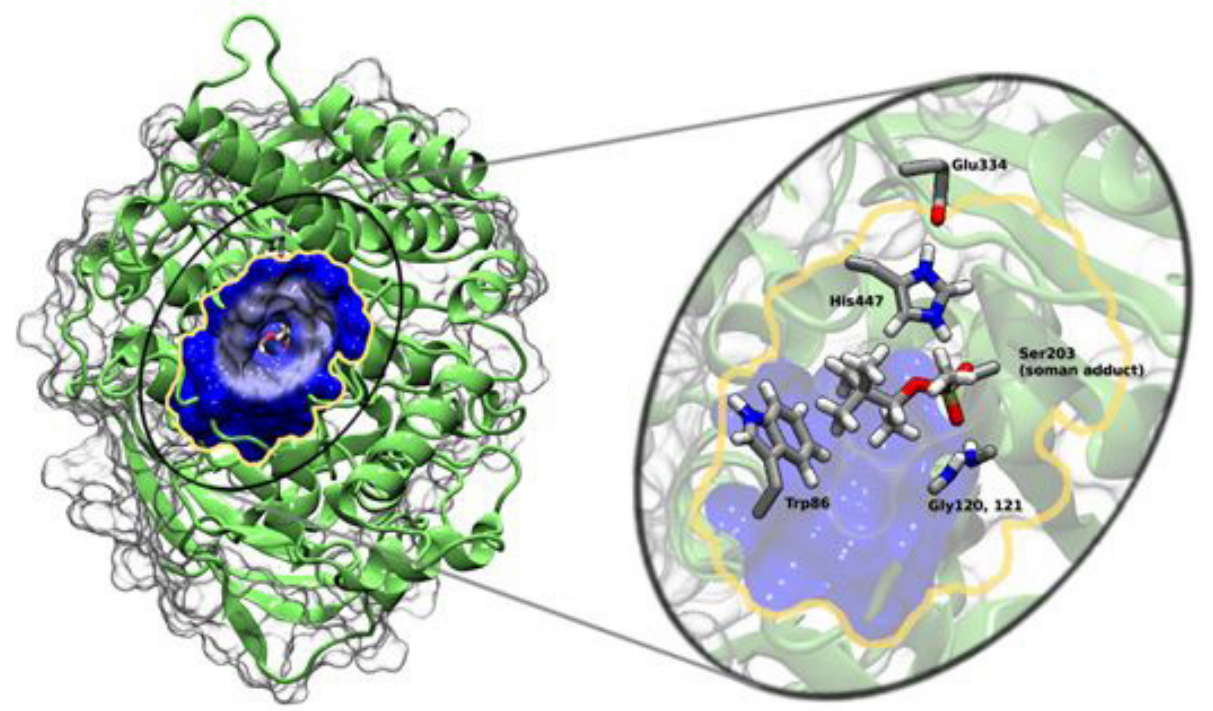

Figure 7: Human AChE structure showing the gorge entrance and important active site amino acid residues [14]

Direct intervention attempts to reactivate the TF-adducted AChE through the use of strong nucleophiles, such as oximes. These compounds act to remove the TF from the enzyme active site, thereby restoring enzymatic function. The active site of AChE consists of a catalytic triad (Ser203, His447, Glu334, human sequence) at the bottom of the $20 \AA$ deep gorge [14-19].

In the absence of trichlorfon, the enzyme catalyzes the hydrolysis reaction so that the AChE concentration decreases, meaning that the number of $h+$ cations increases as the acid is generated, and it recombines with the COOH- on the surface itself. The active site (Ser203, Glu334, His447) of AChE not to close to the TQDs. The interactions between charges on TQDs surface will change 
dramatically. Therefore, recombination will happen less and the intensity of fluorescent emission in this case is not strong $\left(\mathrm{I}_{1}\right)$.

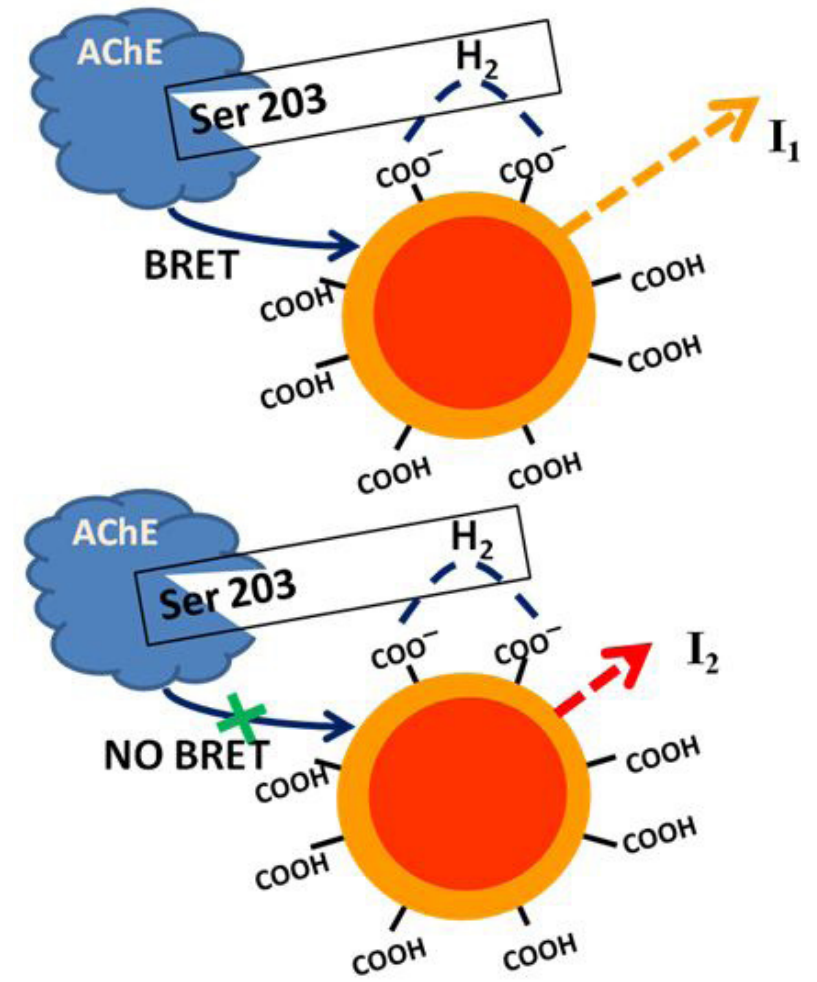

Figure 8: Scheme of the bioluminescence resonance energy transfer (BRET) model

In reverse, when TF was added to the mixture, the AChE was inhibited, the amount of $h+$ ions is almost unchanged, because no hydrolysis reaction occurred. Thus, a higher number of enzymes in the region of 2 to $9 \mathrm{~nm}$ surrounded on the surface TQDs. In the presence of $h+$ cations, the carboxylic acids on the TQDs bound the metal ions and form complexes with the Ser203, Glu334, His447 (active site) on the AChE [17]. BRET took place and produced light emission from the TQDs. The cleaved product displayed a slightly higher (18\%) luminescent emission than the TQDs themselves [4] (Figure 8).

Besides, the trichlorfon molecular might interact with the activity centre of AChE preventing acetic acid production. In contrast, if the number of cation $(h+)$ generated by acetic acid ( $\mathrm{pH}$ decreased) were higher, the $h+$ could capture the electron $(e-)$ on the surface of the TQDs that caused the decrease in $e-h$ recombination in TQDs and the PL quenching phenomena [5,8]. For quantum dots passivity with an inorganic capping layer, the TQDs cores are often assumed to be isolated from the outside environment, leading to the expectation that the TQDs photoluminescence should not be affected by external factors [20].

The result is increased fluorescence intensity $\left(\mathrm{I}_{2}>\mathrm{I}_{1}\right)$.

\section{Conclusion}

$\mathrm{CdZnSe/ZnS} \mathrm{TQDs} \mathrm{that} \mathrm{emit} \mathrm{in} \mathrm{the} \mathrm{visible} \mathrm{region} \mathrm{while} \mathrm{having} \mathrm{a} \mathrm{large} \mathrm{enough} \mathrm{surface} \mathrm{area} \mathrm{to} \mathrm{conjugate} \mathrm{with} \mathrm{biological} \mathrm{agents} \mathrm{have}$ been successfully fabricated. Based on the bioluminescence of the TQDs-AChE system and its BRET, trichlorfon was detected with the concentration of 0.1-5.0 ppm.

\section{Acknowledgment}

The authors thank the National Key Laboratory for Electronic Materials and Devices-IMS for the supplying the facilities. We sincerely thank Academician Professor Nguyen Van Hieu for his judicious discussions and supports.

\section{References}

1. Chen A, Du D, Lin Y (2012) Highly Sensitive and Selective Immuno-Capture/Electrochemical Assay of Acetylcholinesterase Activity in Red Blood Cells: A Biomarker of Exposure to Organophosphorus Pesticides and Nerve Agents, Environ Sci Technol 46: 1828-33.

2. Mercey G, Jean L, Renard PY, Verdelet T, Renou J, et al. (2012) Reactivators of Acetylcholinesterase Inhibited by Organophosphorus Nerve Agents. Acc Chem Res 45: 756-66.

3. Morris-Cohen AJ, Donakowski MD, Knowles KE, Weiss EA (2010) The Effect of a Common Purification Procedure on the Chemical Composition of the surfaces of CdSe Quantum Dots Synthesized with Trioctylphosphine Oxide. J Phys Chem 114: 897-906.

4. Zimmer JP (2006) Quantum dot - Based nanomaterials for Biological Imaging. MIT, USA.

5. Han HS (2012) Development of Fluorescent Semi-conductor Nanocrystal Conjugates for in vitro and in vivo Imaging Applications. MIT, USA. 
6. Zimmer JP (2006) Quantum dot - Based nanomaterials for Biological Imaging. MIT, USA.

7. Periasamy AP, Umasankar Y, Chen SM (2009) Nanomaterials - Acetylcholinesterase Enzyme Matrices for Organophosphorus Pesticides Electrochemical sensors. Sens 9: 4034-55.

8. Dong B, Cao L, Su G, Liu W (2010) Facile Synthesis of Highly Luminescent UV-Blue Emitting ZnSe/ZnS Core/Shell quantum dots by a two-step method. Chem Commun 46: 7331-3.

9. Paek K, Chung S, Choa C-H, Kim BJ (2011) Fluorescent and pH-responsive diblock copolymer-coated core-shell CdSe/ZnS particles for a color-displaying, ratiometric $\mathrm{pH}$ sensor. Chem Commun 47: 10272-4.

10. Luong JH, Male KB, Glennon JD (2008) Biosensor technology: Technology push versus market pull. Biotech Adv 26: $492-500$.

11. Wang JH, Liu TC, Cao YC, Hua XF, Wang HQ, et al. (2007) Fluorescence resonance energy transfer between FITC and water-soluble CdSe/ZnS quantum dots. Colloids Surf 302: 168-73.

12. Liu D, Chen W, Wei J, Li X, Wang Z, et al. (2012) A Highly Sensitive, Dual-Readout Assay Based on Gold Nanoparticles for Organophosphorus and Carbamate Pesticides. Anal. Chem 84: 4185-91.

13. Kloepfer JA, Cohen N, Nadeau JL (2004) FRET between CdSe Quantum Dots in Lipid Vesicles and Water- and Lipid-soluble Dyes. J Phys Chem 108: 17042-9. 14. Kim GB, Kim YP (2012) Analysis of protease activity using quantum dots and resonance energy transfer. Theranostics 2: 127-38.

15.Krauss L (2011) Nanoparicles having continuous photoluminescence. US Patent Application Publication, US 2011/0031452A1.

16. Zhang K1, Mei Q, Guan G, Liu B, Wang S, et al. (2010) Ligand Replacement-Induced Fluorescence Switch of Quantum Dots for Ultrasensitive Detection of Organophosphorothioate Pesticides. Anal. Chem 82: 8579-9588.

17. Frasco MF, Chaniotakis N (2009) Semiconductor Quantum Dots in Chemical Sensors and Biosensors. Sensors 9: $7266-86$.

18. Pohanka M, Jun D, Kalasz H, Kuca K (2013) Cholinesterases in Biorecognition and Biosensors Construction: A Review. Analytical Letters 46: $1849-68$.

19. Rosenthal SJ, Chang JC, Kovtun O, McBride JR, Tomlinson ID (2011) Biocompatible Quantum Dots for Biological Applications. Chem. Bio 18: 10-24.

20. García-Fernández J, Trapiella-Alfonso L, Costa-Fernández JM, Pereiro R, Sanz-Medel A (2014) A Quantum Dot-Based Immunoassay for Screening of Tetracyclines in Bovine Muscle. J Agric Food Chem 62: 1733-40.

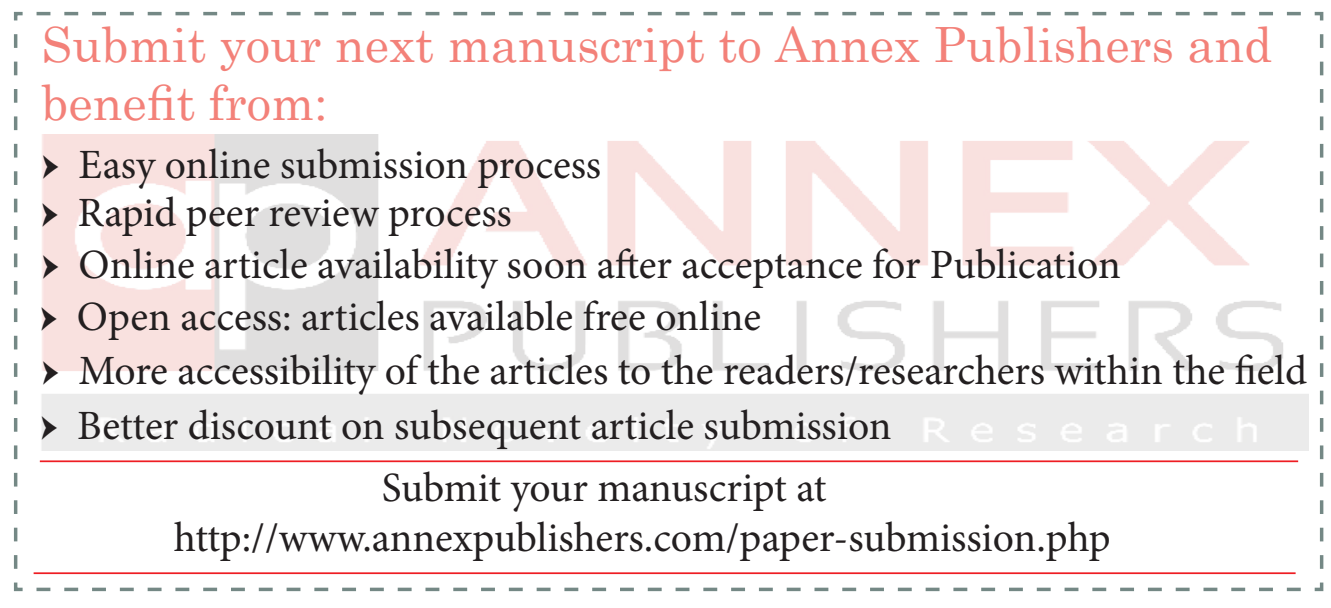

